Research Article

\title{
Stability of Stochastic Differential Delay Systems with Delayed Impulses
}

\author{
Yanlei Wu \\ Department of Mathematics, Anhui Polytechnic University, Wuhu, Anhui 241000, China \\ Correspondence should be addressed to Yanlei Wu; ylwu@ahpu.edu.cn
}

Received 18 November 2013; Accepted 3 March 2014; Published 31 March 2014

Academic Editor: Yiming Ding

Copyright ( 2014 Yanlei Wu. This is an open access article distributed under the Creative Commons Attribution License, which permits unrestricted use, distribution, and reproduction in any medium, provided the original work is properly cited.

\begin{abstract}
We investigate the stability of stochastic delay differential systems with delayed impulses by Razumikhin methods. Some criteria on the $p$ th moment and almost sure exponential stability are obtained. It is shown that an unstable stochastic delay system can be successfully stabilized by delayed impulses. Moreover, it is also shown that if a continuous dynamic system is stable, then, under some conditions, the delayed impulses do not destroy the stability of the systems. The effectiveness of the proposed results is illustrated by two examples.
\end{abstract}

\section{Introduction}

Impulsive dynamical systems have attracted considerable interest in science and engineering in recent years because they provide a natural framework for mathematical modeling of many real world problems where the reactions undergo abrupt changes [1-3]. These systems have found important applications in various fields, such as control systems with communication constraints [4], sampled-data systems $[5,6]$, and mechanical systems [7]. On the other hand, impulsive control based on impulsive systems can provide an efficient way to deal with plants that cannot endure continuous control inputs [3]. In recent years, the impulsive control theory has been generalized from deterministic systems to stochastic systems and has been shown to have wide applications [8].

Stability is one of the most important issues in the study of impulsive stochastic delay differential systems (see e.g., [915]). Particularly, under condition $E V\left(\varphi(0)+I_{k}(\varphi, t), t\right) \leq$ $\rho_{1 k} E V\left(x, t^{-}\right), t=t_{k}$, the $p$ th moment exponential and almost sure exponential stability were investigated in [1214]. In $[12,13]$, the authors show that unstable continuous dynamic systems can be stabilized by impulses. The condition $\rho_{1 k}<1$ is assumed in [12] for any $k \in \mathbb{N}$, which is loosen in [13]. More recently, the condition $\rho_{1 k}<1$ is proved unnecessary when continuous dynamic systems are stable in [14].
In most of recent research results, the impulses are usually assumed to take the following form: $\Delta X\left(t_{k}\right)=X\left(t_{k}^{+}\right)-$ $X\left(t_{k}^{-}\right)=I_{k}\left(X\left(t_{k}^{-}\right), t_{k}\right)$, which indicates the state jump at the impulse time. However, time delays inevitably occurred in the transmission of the impulsive information. Hence, input delays should be considered (see e.g., $[5,16])$. In the context of stability of deterministic differential equations with delayed impulses, there have appeared several results in the literature (see e.g., [17-19]). For example, in [17], the asymptotic stability is investigated for a class of delay-free autonomous systems with the impulses of $\Delta X\left(t_{k}^{+}\right)=C_{1 k} X\left(\left(t_{k}-d_{k}\right)^{-}\right)$, and a sufficient asymptotic stability condition is proposed involving the sizes of impulse input delays. In [19], Chen and Zheng considered more general impulses taking the form $\Delta X\left(t_{k}^{+}\right)=I_{k}\left(X\left(t_{k}^{-}\right), X\left(\left(t_{k}-d_{k}\right)^{-}\right)\right)$and obtained some criteria of exponential stability for nonlinear time-delay systems with delayed impulse effects.

However, most of the existing results of the stability for systems with delayed impulses were considered for the deterministic differential systems. It is noticed that many real world systems are disturbed by stochastic factors. Therefore, it seems interesting to study the stability of stochastic delay differential systems with delayed impulses. Recently, the exponential stability is investigated for impulsive stochastic functional differential system in [20], and exponential stability and uniform stability in terms of two measures 
were obtained for stochastic differential systems with delayed impulses. Motivated by the above works, the aim of this paper is to study $p$ th moment and almost sure exponential stability of a stochastic delay differential system with delayed impulses. It is shown that an unstable stochastic delay system can be successfully stabilized by delayed impulses. Moreover, it is also shown that if a continuous dynamic system is stable, then, under some conditions, the delayed impulses do not destroy the stability of the systems. Our results can generalize some existing results in $[20,21]$.

The paper is organized as follows. In Section 2, we introduce the notations and definitions. We establish several stability criteria for stochastic differential delay systems with delayed impulses in Section 3. In Section 4, two examples are given to illustrate the effectiveness of our results.

\section{Preliminaries}

Throughout this paper, let $(\Omega, \mathscr{F}, P)$ be a complete probability space with some filtration $\left\{\mathscr{F}_{t}\right\}_{t \geqslant 0}$ satisfying the usual conditions (i.e., the filtration is increasing and right continuous while $\mathscr{F}_{0}$ contains all $P$ null sets $)$. Let $B=(B(t), t \geq 0)$ be an $m$-dimensional $\mathscr{F}_{t}$-adapted Brownian motion.

For $x \in \mathbb{R}^{d},|x|$ denotes the Euclidean norm of $x$. For $-\infty<a<b<\infty$, we say that a function from $[a, b]$ to $\mathbb{R}^{d}$ is piecewise continuous, if the function has at most a finite number of jumps discontinuous on $(a, b]$ and are continuous from the right for all points in $[a, b)$. Given $r>0$, $P C\left([-r, 0] ; \mathbb{R}^{d}\right)$ denotes the family of piecewise continuous functions from $[-r, 0]$ to $\mathbb{R}^{d}$ with norm $\|\varphi\|_{r}=\sup _{-r \leq \theta \leq 0} \varphi(\theta)$. For $p \geq 1$ and $t \geq t_{0}$, let $L_{\mathscr{F}_{t}}^{p}\left([-r, 0] ; \mathbb{R}^{d}\right)$ be the family of $\mathscr{F}_{t^{-}}$ adapted and $P C\left([-r, 0] ; \mathbb{R}^{d}\right)$-valued random variables $\varphi$ such that $E\|\varphi\|_{r}^{p}<\infty$. Let $\mathbb{N}=1,2, \ldots$ and $\mathbb{R}^{+}=[0,+\infty)$.

In this paper, we consider the following stochastic delay differential systems with delayed impulses:

$$
\begin{gathered}
d X(t)=f\left(X_{t}, t\right) d t+g\left(X_{t}, t\right) d B(t), \\
t \neq t_{k}, \quad t \geq t_{0} ; \\
\Delta X\left(t_{k}\right)=X\left(t_{k}\right)-X\left(t_{k}^{-}\right)=I_{k}\left(X\left(t^{-}\right), X\left(t-d_{k}\right)^{-}\right), \\
k \in \mathbb{N} ; \\
X_{t_{0}}=\xi\left(t_{0}+\theta\right), \quad-\tau \leq \theta \leq 0,
\end{gathered}
$$

where $\left\{t_{k}, k \in \mathbb{N}\right\}$ is a strictly increasing sequence such that $t_{k} \rightarrow \infty$ as $k \rightarrow \infty ;\left\{d_{k} \geq 0, k \in \mathbb{N}\right\}$ are the impulsive input delays satisfying $d=\max _{k} d_{k}$ and $\tau=\max \{r, d\}$. $X_{t}$ is defined by $X_{t}(\theta)=X(t+\theta),-r \leq \theta \leq 0$. Let $X_{t^{-}}(\theta)=X\left((t+\theta)^{-}\right),-r \leq$ $\theta \leq 0$, where $X\left(t^{-}\right)=\lim _{s \rightarrow t^{-}} X(s)$. The mappings $I: \mathbb{R}^{d} \times$ $P C\left([-r, 0] ; \mathbb{R}^{d}\right) \rightarrow \mathbb{R}^{d}, f: P C\left([-r, 0] ; \mathbb{R}^{d}\right) \times \mathbb{R}^{+} \rightarrow \mathbb{R}^{d}$, and $g: P C\left([-r, 0] ; \mathbb{R}^{d}\right) \times \mathbb{R}^{+} \rightarrow \mathbb{R}^{d \times m}$ are all Borel-measurable functions. For simplicity, denote $V(x(t), t)$ by $V(t)$.

As a standing hypothesis, we assume that $f, g$, and $I$ are assumed to satisfy necessary assumptions so that, for any $\xi \in L_{\mathscr{F}_{t}}^{p}\left([-\tau, 0] ; \mathbb{R}^{d}\right)$, system (1) has a unique global solution, denoted by $X(t ; \xi)$, and, moreover, $X(t ; \xi) \in L_{\mathscr{F}_{t}}^{p}\left([-r, 0] ; \mathbb{R}^{d}\right)$. In addition, we assume that $f(0, t) \equiv 0, g(0, t) \equiv 0$ and $I_{k}(0,0) \equiv 0$, for all $t \geq t_{0}, k \in \mathbb{N}$; then system (1) admits a trivial solution $X(t) \equiv 0$. Moreover, we make the following assumptions on system (1).

$\left(\mathrm{A}_{1}\right)$ There is a constant $L>0$, such that

$$
\begin{array}{r}
E\left(\left|f\left(X_{t}, t\right)\right|^{p}+\left|g\left(X_{t}, t\right)\right|^{p}\right)<L \sup _{-r \leq \theta \leq 0} E|X(t)|^{p}, \\
t \geq t_{0} .
\end{array}
$$

$\left(\mathrm{A}_{2}\right)$ There exist nonnegative bounded sequences $\left\{h_{1 k}\right\}$ and $\left\{h_{2 k}\right\}$ such that

$$
\left|I_{k}(x, y)\right| \leq h_{1 k}|x|+h_{2 k}|y|, \quad k \in \mathbb{N} .
$$

Set $\bar{h}=\sup _{k}\left(h_{1 k}+h_{2 k}\right)$.

Let $C^{2,1}\left(\mathbb{R}^{d} \times\left[t_{0}-r, \infty\right) ; \mathbb{R}^{+}\right)$denote the family of all nonnegative functions $V(x, t)$ on $\mathbb{R}^{d} \times\left[t_{0}-r, \infty\right)$ that are continuously twice differentiable in $x$ and once in $t$. For each $V \in C^{2,1}\left(\mathbb{R}^{d} \times\left[t_{0}-r, \infty\right) ; \mathbb{R}^{+}\right)$, define an operator $\mathscr{L} V:$ $P C\left([-r, 0] ; \mathbb{R}^{d}\right) \times \mathbb{R}^{+} \rightarrow \mathbb{R}^{d}$ for system (1) by

$$
\begin{aligned}
\mathscr{L} V\left(X_{t}, t\right)= & V_{t}(x, t)+V_{x}(x, t) f\left(X_{t}, t\right) \\
& +\frac{1}{2} \operatorname{trace}\left[g^{T}\left(X_{t}, t\right) V_{x x}(x, t) g\left(X_{t}, t\right)\right],
\end{aligned}
$$

where

$$
\begin{gathered}
V_{t}(x, t)=\frac{\partial V(x, t)}{\partial t} \\
V_{x}(x, t)=\left(\frac{\partial V(x, t)}{\partial x_{1}}, \ldots, \frac{\partial V(x, t)}{\partial x_{d}}\right), \\
V_{x x}(x, t)=\left(\frac{\partial^{2} V(x, t)}{\partial x_{i} \partial x_{j}}\right)_{d x d} .
\end{gathered}
$$

The purpose of this paper is to discuss the stability of system (1). Let us begin with the following definition.

Definition 1. The trivial solution of system (1) is said to be as follows.

(1) $p$ th moment exponentially stable, if, for any initial data $\xi \in L_{\mathscr{F}_{t_{0}}}^{p}\left([-r, 0] ; \mathbb{R}^{d}\right)$, the solution $X(t)$ satisfies

$$
E|X(t)|^{p} \leq C E\|\xi\|^{p} e^{-\lambda\left(t-t_{0}\right)}
$$

or, equivalently,

$$
\limsup _{t \rightarrow \infty} \frac{1}{t} \log E|X(t)|^{p} \leq-\lambda,
$$

where $\lambda$ and $C$ are positive constants independent of $t_{0}$.

(2) Almost sure exponentially stable, if the solution $X(t)$ satisfies

$$
\limsup _{t \rightarrow \infty} \frac{1}{t} \log |X(t)|<-\lambda
$$

for any initial data $\xi \in L_{\mathscr{F}_{t_{0}}}^{p}\left([-r, 0] ; \mathbb{R}^{d}\right)$ and $\lambda>0$. 


\section{Main Results}

Before establishing the main results, we derive the following lemma, which is useful to present the main results.

Lemma 2. Let assumptions $\left(A_{1}\right)$ and $\left(A_{2}\right)$ hold. Suppose that $\inf _{k \in \mathbb{N}}\left\{t_{k}-t_{k-1}\right\}=\beta_{1}$ and $\left(l_{1}-1\right) \beta_{1}<d \leq l_{1} \beta_{1}$ for some positive integer $l_{1}$. Then

$$
E|X(t)|^{p} \leq K_{1} E\|\xi\|_{\tau}^{p}, \quad t \in\left[t_{0}-\tau, t_{0}+d\right],
$$

where $K_{1}=3^{l_{1}(p-1)}(1+\bar{h})^{l_{1}} e^{3^{p-1} L\left(d^{p}+d^{p / 2}\right)}$.

Proof. Since $\left(l_{1}-1\right) \beta_{1}<d \leq l_{1} \beta_{1}$, the maximum number of impulsive times on the interval $\left(t_{0}, t_{0}+d\right]$ is $l_{1}$. Suppose that the impulsive instants on $\left(t_{0}, t_{0}+d\right]$ are $t_{i}, 1 \leq i \leq m \leq l_{1}$. For $t \in\left(t_{0}, t_{1}\right)$, using $\left(\mathrm{A}_{1}\right)$, we have

$$
\begin{aligned}
& E|X(t)|^{p}=E\left|\xi(0)+\int_{t_{0}}^{t} f\left(X_{s}, s\right) d s+\int_{t_{0}}^{t} g\left(X_{s}, s\right) d B(s)\right|^{p} \\
& \leq 3^{p-1}\left[E\|\xi\|_{\tau}^{p}+\left(t-t_{0}\right)^{p-1} E \int_{t_{0}}^{t}\left|f\left(X_{s}, s\right)\right|^{p} d s\right. \\
& \left.+\left(t-t_{0}\right)^{(p-2) / 2} E \int_{t_{0}}^{t}\left|g\left(X_{s}, s\right)\right|^{p} d s\right] \\
& \leq 3^{p-1}\left[E\|\xi\|_{\tau}^{p}+L\left(t-t_{0}\right)^{p-1} \int_{t_{0}}^{t} E \sup _{t_{0}-r \leq u \leq s}|X(u)|^{p} d s\right. \\
& \left.+L\left(t-t_{0}\right)^{(p-2) / 2} \int_{t_{0}}^{t} E \sup _{t_{0}-r \leq u \leq s}|X(u)|^{p} d s\right] \\
& \leq 3^{p-1} E\|\xi\|_{\tau}^{p}+3^{p-1} L \\
& \times\left[\left(t-t_{0}\right)^{p-1}+\left(t-t_{0}\right)^{(p-2) / 2}\right] \\
& \times \int_{t_{0}}^{t} E \sup _{t_{0}-r \leq u \leq s}|X(u)|^{p} d s
\end{aligned}
$$

which implies

$$
\begin{aligned}
E \sup _{t_{0}-r \leq s \leq t}|X(s)|^{p} \leq & 3^{p-1} E\|\xi\|_{\tau}^{p}+3^{p-1} L\left(d^{p-1}+d^{(p-2) / 2}\right) \\
& \times \int_{t_{0}}^{t} E \sup _{t_{0}-r \leq u \leq s}|X(u)|^{p} d s .
\end{aligned}
$$

Using the Gronwall inequality, it follows that

$$
\begin{array}{r}
E \sup _{t_{0}-r \leq s \leq t}|X(s)|^{p} \leq 3^{p-1} E\|\xi\|_{\tau}^{p} e^{3^{p-1} L\left(d^{p-1}+d^{(p-2) / 2}\right)\left(t-t_{0}\right)}, \\
t \in\left(t_{0}, t_{1}\right) .
\end{array}
$$

According to $\left(A_{2}\right)$, we get

$$
\begin{aligned}
\left|X\left(t_{1}\right)\right| & =\left|X\left(t_{1}^{-}\right)+I_{k}\left(X\left(t_{1}^{-}\right), X\left(\left(t_{1}-d_{1}\right)^{-}\right)\right)\right| \\
& \leq\left|X\left(t_{1}^{-}\right)\right|+h_{11}\left|X\left(t_{1}^{-}\right)\right|+h_{12}\left|X\left(\left(t_{1}-d_{1}\right)^{-}\right)\right| .
\end{aligned}
$$

It follows that

$$
E\left|X\left(t_{1}\right)\right|^{p} \leq 3^{p-1}(1+\bar{h}) E\|\xi\|_{\tau}^{p} e^{3^{p-1} L\left(d^{p-1}+d^{(p-2) / 2}\right)\left(t-t_{0}\right)} .
$$

Hence,

$$
\begin{array}{r}
E|X(t)|^{p} \leq 3^{p-1}(1+\bar{h}) E\|\xi\|_{\tau}^{p} e^{3^{p-1} L\left(d^{p-1}+d^{(p-2) / 2}\right)\left(t_{1}-t_{0}\right),} \\
t \in\left[t_{0}-\tau, t_{1}\right] .
\end{array}
$$

Repeating the above argument gives that, for $t \in\left[t_{0}-\tau, t_{m}\right]$,

$$
E|X(t)|^{p} \leq 3^{l_{1}(p-1)}(1+\bar{h})^{l_{1}} E\|\xi\|_{\tau}^{p} e^{3^{p-1} L\left(d^{p-1}+d^{(p-2) / 2}\right)\left(t_{m}-t_{0}\right)} .
$$

Since there are no impulses on $\left(t_{m}, t_{0}+d\right]$, we obtain

$$
\begin{array}{r}
E|X(t)|^{p} \leq 3^{l_{1}(p-1)}(1+\bar{h})^{l_{1}} E\|\xi\|_{\tau}^{p} e^{3^{p-1} L\left(d^{p-1}+d^{(p-2) / 2}\right) d}, \\
t \in\left[t_{0}-\tau, t_{0}+d\right] .
\end{array}
$$

This completes the proof.

When the continuous dynamics in system (1) is unstable, the following theorem shows that the system (1) can be stabilized by the delayed impulses.

Theorem 3. Let the assumptions in Lemma 2 hold. Assume that there exist positive constants $c_{1}, c_{2}, \gamma_{1}$, and $\lambda$ and $p \geq 1$ such that

$$
\begin{aligned}
& \left(\mathrm{H}_{1}\right) c_{1}|x|^{p} \leq V(x, t) \leq c_{2}|x|^{p} \\
& \left(\mathrm{H}_{2}\right) \text { for } t \in\left[t_{k-1}, t_{k}\right), k \in \mathbb{N}
\end{aligned}
$$

$$
E \mathscr{L} V(\varphi(\theta), t) \leq \gamma_{1} E V(\varphi(0), t)
$$

provided that $\varphi \in L_{\mathscr{F}_{t}}^{p}\left([-r, 0] ; \mathbb{R}^{d}\right)$ satisfies $E V(\varphi(\theta), t+\theta) \leq$ $q E V(\varphi(0), t), \theta \in[-r, 0]$;

$\left(\mathrm{H}_{3}\right)$ there exist nonnegative constant sequences $\rho_{1 k}, \rho_{2 k}$, and $\eta_{k}$ such that

$$
\begin{aligned}
E V\left(t, x+I_{k}(x, y)\right) \leq & \rho_{1 k} \eta_{k} E V\left(x, t^{-}\right) \\
& +\rho_{2 k} \eta_{k} E V\left(y,\left(t-d_{k}\right)^{-}\right), \quad t=t_{k},
\end{aligned}
$$

where $\prod_{k=1}^{\infty} \eta_{k}<\infty$;

$\left(\mathrm{H}_{4}\right)$ let $\gamma=\sup _{m \in \mathbb{N}}\left\{\rho_{1 m}+\rho_{2 m} e^{\lambda d}\right\}, q \geq\left(e^{\lambda r} / \gamma\right)$ and $\gamma<$ $e^{-\left(\gamma_{1}+\lambda\right) \beta_{2}}$, where $\beta_{2}=\sup _{k \in \mathbb{N}}\left\{t_{k}-t_{k-1}\right\}<\infty$. 
Then the trivial solution of system (1) is pth moment exponentially stable.

Proof. Define $W(t)=e^{\lambda\left(t-t_{0}-d\right)} V(t)$. From Itô's differential formula, we have

$$
d W(t)=\mathscr{L} W(t)+e^{\lambda\left(t-t_{0}-d\right)} V_{x}\left(X_{t}, t\right) g\left(X_{t}, t\right) d B(t),
$$

for $t \in\left[t_{k-1}, t_{k}\right), k \in \mathbb{N}$. It is easy to calculate that

$$
\mathscr{L} W(t)=\lambda e^{\lambda\left(t-t_{0}-d\right)} V(t)+e^{\lambda\left(t-t_{0}-d\right)} \mathscr{L} V(t) .
$$

Let $\Delta t>0$ be small enough such that $t+\Delta t \in\left(t_{k-1}, t_{k}\right)$, then

$$
E W(t+\Delta t)-E W(t)=\int_{t}^{t+\Delta t} E \mathscr{L} W(s) d s,
$$

which implies that

$$
D^{+} E W(t)=E \mathscr{L} W(t), \quad t \in\left[t_{k-1}, t_{k}\right), k \in \mathbb{N} .
$$

In view of Lemma 2 and $\left(\mathrm{H}_{1}\right)$, we obtain

$$
E W(t) \leq c_{2} K_{1} E\|\xi\|^{p} \leq \gamma M, \quad t \in\left[t_{0}-\tau, t_{0}+d\right],
$$

where $M=c_{2} K_{1} E\|\xi\|^{p} / \gamma$. In the following, we will prove

$$
E W(t) \leq M, \quad t \geq t_{0}+d .
$$

We first show that

$$
E W(t) \leq M, \quad t \in\left[t_{0}+d, t_{1}\right) .
$$

Suppose that it is not true; then there exist some $t \in\left(t_{0}+d, t_{1}\right)$ such that $E W(t)>M$. Set $t^{*}=\inf \left\{t \in\left[t_{0}+d, t_{1}\right): E W(t)>\right.$ $M$; ; we have $t^{*} \in\left(t_{0}+d, t_{1}\right)$ and $E W\left(t^{*}\right)=M$. Let $t_{*}=$ $\sup \left\{t \in\left[t_{0}+d, t^{*}\right): E W(t) \leq \gamma M\right\}$. For $t \in\left[t_{*}, t^{*}\right]$, we see that

$$
E W(t) \geq \gamma M \geq \gamma E W(t+\theta), \quad \theta \in[-r, 0] .
$$

Hence,

$$
\begin{aligned}
E V(X(t), t) & \geq \gamma e^{-\lambda r} E V(X(t+\theta), t+\theta) \\
& \geq \frac{1}{q} E V(X(t+\theta), t+\theta), \quad \theta \in[-r, 0] .
\end{aligned}
$$

Combining this with $\left(\mathrm{H}_{2}\right)$, we obtain that, for $t \in\left[t_{*}, t^{*}\right]$,

$$
D^{+} E W(t) \leq e^{\lambda\left(t-t_{0}-d\right)}\left(\gamma_{1}+\lambda\right) E V(t)=\left(\gamma_{1}+\lambda\right) E W(t) .
$$

So, we derive that

$$
E W\left(t^{*}\right) \leq E W\left(t_{*}\right) e^{\left(\gamma_{1}+\lambda\right)\left(t^{*}-t_{*}\right)} \leq \gamma M e^{\left(\gamma_{1}+\lambda\right) \beta_{2}}<M .
$$

It is a contradiction; therefore, (26) holds for $t \in\left(t_{0}+d, t_{1}\right)$.
Now, we assume that $E W(t) \leq \prod_{k=1}^{m-1} \eta_{k} M, t \in\left[t_{m-1}, t_{m}\right)$, $m \in \mathbb{N}$. We will show that

$$
E W(t) \leq \prod_{k=1}^{m} \eta_{k} M, \quad t \in\left[t_{m}, t_{m+1}\right) .
$$

By $\left(\mathrm{H}_{3}\right)$, we derive that

$$
\begin{aligned}
E W\left(t_{m}\right)= & e^{\lambda\left(t_{m}-t_{0}-d\right)} E V\left(t_{m}\right) \leq e^{\lambda\left(t_{m}-t_{0}-d\right)} \\
& \times\left(\rho_{1 m} \eta_{m} E V\left(t_{m}^{-}\right)+\rho_{2 m} \eta_{m} E V\left(\left(t_{m}-d_{m}\right)^{-}\right)\right) \\
\leq & \rho_{1 m} \eta_{m} E W\left(t_{m}^{-}\right)+\rho_{2 m} e^{\lambda d} \eta_{m} E W\left(\left(t_{m}-d_{m}\right)^{-}\right) \\
\leq & \left(\rho_{1 m}+\rho_{2 m} e^{\lambda d}\right) \prod_{k=1}^{m} \eta_{k} M \\
= & \gamma \prod_{k=1}^{m} \eta_{k} M .
\end{aligned}
$$

Now, we assume that (31) is not true. Set $t^{*}=\inf \{t \in$ $\left.\left[t_{m}, t_{m+1}\right): E W(t)>\prod_{k=1}^{m} \eta_{k} M\right\}$; then we have $t^{*} \in\left(t_{m}, t_{m+1}\right)$ and $E W\left(t^{*}\right)=\prod_{k=1}^{m} \eta_{k} M$. Let $t_{*}=\sup \left\{t \in\left[t_{m}, t^{*}\right): E W(t) \leq\right.$ $\left.\gamma \prod_{k=1}^{m} \eta_{k} M\right\}$. For $t \in\left[t_{*}, t^{*}\right]$, we have

$$
E W(t) \geq \gamma \prod_{k=1}^{m} \eta_{k} M \geq \gamma E W(t+\theta), \quad \theta \in[-r, 0] .
$$

Hence,

$$
\begin{aligned}
E V(X(t), t) & \geq \gamma e^{-\lambda r} E V(X(t+\theta), t+\theta) \\
& \geq \frac{1}{q} E V(X(t+\theta), t+\theta), \quad \theta \in[-r, 0] .
\end{aligned}
$$

This yields that $D^{+} E W(t) \leq\left(\gamma_{1}+\lambda\right) E W(t), t \in\left[t_{*}, t^{*}\right]$. Therefore,

$$
\begin{aligned}
E W\left(t^{*}\right) & \leq E W\left(t_{*}\right) e^{\left(\gamma_{1}+\lambda\right)\left(t^{*}-t_{*}\right)} \\
& \leq \gamma \prod_{k=1}^{m} \eta_{k} M e^{\left(\gamma_{1}+\lambda\right) \beta_{2}}<\prod_{k=1}^{m} \eta_{k} M,
\end{aligned}
$$

which leads to a contradiction. Thus, (31) holds.

By mathematical induction, we have

$$
E W(t)<M \prod_{k=1}^{\infty} \eta_{k}, \quad t \geq t_{0} .
$$

This implies that

$$
E|X(t)|^{p}<\frac{M \prod_{k=1}^{\infty} \eta_{k}}{c_{1}} e^{-\lambda\left(t-t_{0}-d\right)}, \quad t \geq t_{0} .
$$

This completes the proof. 
Remark 4. In Theorem 3, the positive constant $\eta_{k}$ is introduced in $\left(\mathrm{H}_{3}\right)$, where $\eta_{k}>1$ and $\eta_{k} \leq 1$ are allowed. As mentioned in [13], the constant $\eta_{k}$ is introduced in $\left(\mathrm{H}_{3}\right)$, which makes it possible to tolerate certain perturbations in the overall impulsive stabilization process; that is, it is not strictly required by Theorem 3 that each impulse contributes to stabilize the system; there can exist some destabilized impulses. Moreover, when $\eta_{3 k-2}=1 / 2, \eta_{3 k-1}=1 / 2, \eta_{3 k}=4$, for $k \in \mathbb{N}$, we have $\prod_{k=1}^{\infty} \eta_{k}<5$ and $\sum_{k=1}^{\infty}\left(\eta_{k}-1\right)=+\infty$. Then, Theorem 3 can be used, but the results in $[20,21]$ cannot be applicable to this case.

In the following theorem, we will show that if the continuous dynamics is stable, then, under some condition, the system is still stable with the delayed impulsive effects.

Theorem 5. Assume that the assumptions in Lemma 2 hold. Suppose that there exist positive constants $c_{1}, c_{2}, \gamma_{2}$, and $\lambda$ and $p \geq 1$ such that

$\left(\mathrm{H}_{1}\right) c_{1}|x|^{p} \leq V(t, x) \leq c_{2}|x|^{p} ;$

$\left(\mathrm{H}_{2}\right)$ for $t \in\left[t_{k-1}, t_{k}\right), k \in \mathbb{N}$ and $V \in C^{2,1}\left(\mathbb{R}^{n} \times\left[t_{0}-\right.\right.$ $\left.r, \infty) ; \mathbb{R}^{+}\right)$,

$$
E \mathscr{L} V(\varphi(\theta), t) \leq-\gamma_{2} E V(\varphi(0), t)
$$

provided that $\varphi \in L_{\mathscr{F}_{t}}^{p}\left([-r, 0] ; \mathbb{R}^{d}\right)$ satisfies $E V(\varphi(\theta), t+\theta) \leq$ $q E V(\varphi(0), t), \theta \in[-r, 0]$;

$$
\begin{aligned}
\left(\mathrm{H}_{3}\right) & E V\left(x+I_{k}(x, y), t\right) \leq \rho_{1 k} E V\left(x, t^{-}\right)+\rho_{2 k} E V(y,(t- \\
& \left.\left.d_{k}\right)^{-}\right), \text {for all } t=t_{k} ; \\
\left(\mathrm{H}_{4}\right) & \sup _{k \in \mathbb{N}}\left\{\left(\rho_{1 k} / \min \left\{q e^{-\lambda r}, e^{\left(\gamma_{2}-\lambda\right) \beta_{1}}\right\}\right)+\rho_{2 k} e^{\lambda d}\right\}<1, \\
& q e^{-\lambda r}>1 \text { and } \gamma_{2}>\lambda .
\end{aligned}
$$

Then the trivial solution of system (1) is pth moment exponentially stable.

Proof. Since $\max _{k \in \mathbb{N}}\left\{\left(\rho_{1 k} / \min \left\{q e^{-\lambda r}, e^{\left(\gamma_{2}-\lambda\right) \beta_{1}}\right\}\right)+\rho_{2 k} e^{\lambda d}\right\}<1$, $q e^{-\lambda r}>1$ and $e^{\left(\gamma_{2}-\lambda\right) \beta_{1}}>1$, there exists a constant $\bar{q}>1$ such that

$$
\begin{array}{r}
1<\bar{q}<\min \left\{q e^{-\lambda r}, e^{\left(\gamma_{2}-\lambda\right) \beta_{1}}\right\}, \frac{\rho_{1 k}}{\bar{q}}+\rho_{2 k} e^{\lambda d} \leq 1, \\
k \in \mathbb{N} .
\end{array}
$$

By Lemma 2 and $\left(\mathrm{H}_{1}\right)$, we have

$$
E W(t) \leq c_{2} K_{1} E\|\xi\|^{p} \leq \frac{1}{\bar{q}} M, \quad t \in\left[t_{0}-\tau, t_{0}+d\right],
$$

where $M=c_{2} \bar{q} K_{1} E\|\xi\|^{p}$. We first show

$$
E W(t) \leq M, \quad t \in\left[t_{0}+d, t_{1}\right] .
$$

This can be verified by a contradiction. Suppose that it is not true, then there exist some $t \in\left[t_{0}+d, t_{1}\right)$ such that $E W(t)>$ $M$. Set $\overline{t^{*}}=\inf \left\{t \in\left[t_{0}+d, t_{1}\right): E W(t) \leq M\right\}$, then $\overline{t^{*}} \in$ $\left(t_{0}+d, t_{1}\right)$. Let $\overline{t_{*}}=\sup \left\{t \in\left[t_{0}+d, \overline{t^{*}}\right): E W(t) \geq(1 / \bar{q}) M\right\}$. For $t \in\left[\overline{t_{*}}, \overline{t^{*}}\right]$, we get

$$
\bar{q} E W(t) \geq M \geq E W(t+\theta), \quad-r \leq \theta \leq 0 .
$$

Hence,

$$
\begin{aligned}
E V(X(t), t) & \geq \frac{1}{\bar{q}} e^{-\lambda r} E V(X(t+\theta), t+\theta) \\
& \geq \frac{1}{q} E V(X(t+\theta), t+\theta), \quad-r \leq \theta \leq 0 .
\end{aligned}
$$

It follows that, for $t \in\left[\overline{t_{*}}, \overline{t^{*}}\right]$,

$$
\begin{aligned}
D^{+} E W(t) & \leq \lambda e^{\lambda\left(t-t_{0}-d\right)} E V(t)-\gamma_{2} e^{\lambda\left(t-t_{0}-d\right)} E V(t) \\
& =\left(\lambda-\gamma_{2}\right) E W(t)<0
\end{aligned}
$$

which yields that $E W\left(\overline{t^{*}}\right) \leq E W\left(\overline{t_{*}}\right)=(1 / \bar{q}) M<M$. This is a contradiction; therefore, (41) holds for $\left[t_{0}+d, t_{1}\right)$.

Now we assume that

$$
E W(t) \leq M, \quad t \in\left[t_{m-1}, t_{m}\right), m \in \mathbb{N} .
$$

We will show that

$$
E W(t) \leq M, \quad t \in\left[t_{m}, t_{m+1}\right) .
$$

In order to do this, we first prove that

$$
E W\left(t_{m}^{-}\right) \leq \frac{1}{\bar{q}} M
$$

Suppose this is not true, then $E W\left(t_{m}^{-}\right)>(1 / \bar{q}) M$. There exist two possible cases as follows.

Case 1. $E W(t)>(1 / \bar{q}) M$, for all $t \in\left[t_{m-1}, t_{m}\right)$. Obviously, for $t \in\left[t_{m-1}, t_{m}\right)$,

$$
\bar{q} E W(t) \geq M \geq E W(t+\theta), \quad-r \leq \theta \leq 0 .
$$

Thus, we can get $D^{+} E W(t) \leq\left(\lambda-\gamma_{2}\right) E W(t)$, which implies that

$$
\begin{aligned}
E W\left(t_{m}^{-}\right) & \leq E W\left(t_{m-1}\right) e^{\left(\lambda-\gamma_{2}\right)\left(t_{m}-t_{m-1}\right)} \\
& \leq E W\left(t_{m-1}\right) e^{\left(\lambda-\gamma_{2}\right) \beta_{1}}<\frac{1}{\bar{q}} M .
\end{aligned}
$$

This is a contradiction.

Case 2. There exist some $s \in\left[t_{m-1}, t_{m}\right)$ such that $E W(s) \leq$ $(1 / \bar{q}) M$. In this case, set $\bar{t}=\sup \left\{t \in\left[t_{m-1}, t_{m}\right): E W(t)<\right.$ $(1 / \bar{q}) M\}$; then $E W(\bar{t})=(1 / \bar{q}) M$. Since, for $t \in\left[\bar{t}, t_{m}\right)$,

$$
\bar{q} E W(t) \geq M \geq E W(t+\theta), \quad-r \leq \theta \leq 0,
$$

it follows that $D^{+} E W(t) \leq 0$, which gives $E W\left(t_{m}^{-}\right) \leq E W(\bar{t})=$ $(1 / \bar{q}) M$. This is also a contradiction. 
Hence, (47) holds. In the following equation, we will show that $E W\left(t_{m}\right) \leq M$. In view of $\left(\mathrm{H}_{3}\right)$, we obtain

$$
\begin{aligned}
E W\left(t_{m}\right) & =e^{\lambda\left(t_{m}-t_{0}-d\right)} E V\left(t_{m}\right) \\
& \leq e^{\lambda\left(t_{m}-t_{0}-d\right)}\left(\rho_{1} E V\left(t_{m}^{-}\right)+\rho_{2} E V\left(\left(t_{m}-d_{m}\right)^{-}\right)\right) \\
& \leq \rho_{1} E W\left(t_{m}^{-}\right)+\rho_{2} e^{\lambda d} E W\left(\left(t_{m}-d_{m}\right)^{-}\right) \\
& \leq\left(\frac{\rho_{1}}{\bar{q}}+\rho_{2} e^{\lambda d}\right) M \\
& \leq M
\end{aligned}
$$

We go on proving (46). Suppose that it is not the case; then, there exist some $t \in\left[t_{m}, t_{m+1}\right)$. Set $\overline{t^{*}}=\inf \left\{t \in\left[t_{m}, t_{m+1}\right)\right.$ : $E W(t)>M\}$; then, we have $\overline{t^{*}} \in\left(t_{m}, t_{m+1}\right)$. If $E W(t) \geq$ $(1 / \bar{q}) M$, set $\overline{t_{*}}=t_{m}$; otherwise, set $\overline{t_{*}}=\sup \left\{t \in\left[t_{m}, \overline{t^{*}}\right)\right.$ : $E W(t) \leq(1 / \bar{q}) M\}$. For $t \in\left[\overline{t_{*}}, \overline{t^{*}}\right]$, we derive

$$
\bar{q} E W(t) \geq M \geq E W(t+\theta), \quad-r \leq \theta \leq 0,
$$

which implies that

$$
\begin{aligned}
E V(t) & \geq \frac{1}{\bar{q}} e^{-\lambda r} E V(t+\theta, X(t+\theta)) \\
& \geq \frac{1}{q} E V(t+\theta, X(t+\theta)), \quad-r \leq \theta \leq 0 .
\end{aligned}
$$

It follows that $D^{+} E W(t)<0$ for $t \in\left[\overline{t_{*}}, \overline{t^{*}}\right]$. Consequently, $E W\left(\overline{t^{*}}\right)<E W\left(\overline{t_{*}}\right)$. This is a contradiction. Thus, (46) holds. By mathematical induction, we see that

$$
E W(t) \leq M, \quad t \geq t_{0}-\tau
$$

Then we can get from $\left(\mathrm{H}_{1}\right)$ that

$$
E|X(t)|^{p} \leq \frac{M}{c_{1}} e^{-\lambda\left(t-t_{0}-d\right)}, \quad t \geq t_{0}-\tau
$$

This completes the proof.

Remark 6. When the continuous system in system (1) is stable, the system (1) can always be stable with stabilized impulses. Thus, $\rho_{1 k}+\rho_{2 k}<1$ is permissible in Theorem 5, and only one constraint $q e^{-\lambda r}>1$ is assumed for constant $q$. However, $\rho_{1}+\rho_{2} \geq 1$ and $\rho_{1}+\rho_{2} e^{\tilde{c} \tau}>q$ are necessary in Theorem 3.2 of [20]. Thus, in this aspect, Theorem 5 is more general than the results existing in [20].

The following theorem shows that the trivial solution of system (1) is almost sure exponentially stable, under some additional conditions.

Theorem 7. Suppose that $p \geq 1$ and the conditions in Theorem 3 or Theorem 5 hold. Then, the trivial solution of system (1) is almost sure exponentially stable.
Proof. Using Theorem 3 or Theorem 5, we derive that the trivial solution of system (1) is pth moment exponentially stable. Therefore, there exists a positive constant $C_{1}$ such that

$$
E|X(t)|^{p} \leq C_{1} e^{-\lambda\left(t-t_{0}\right)} .
$$

It is obvious that

$$
\begin{aligned}
& E\left(\sup _{0 \leq s \leq r}|X(t+s)|^{p}\right) \\
& \leq 4^{p-1}\left(E|X(t)|^{p}+E\left(\int_{t}^{t+r}\left|f\left(X_{s}, s\right)\right| d s\right)^{p}\right. \\
& +E\left|\sup _{0 \leq s \leq r} \int_{t}^{t+s} g\left(X_{u}, u\right) d B(u)\right|^{p} \\
& \left.+E\left|\sum_{t \leq t_{k} \leq t+r} I_{k}\left(X\left(t^{-}\right), X\left(t-d_{k}\right)^{-}\right)\right|^{p}\right) .
\end{aligned}
$$

Combining the Hölder inequality with $\left(A_{1}\right)$ and (56) implies that

$$
\begin{aligned}
E \int_{t}^{t+r}\left|f\left(X_{s}, s\right)\right|^{p} d s & \leq L r^{p-1} \int_{t}^{t+r} \sup _{-r \leq \theta \leq 0} E|X(s+\theta)|^{p} d s \\
& \leq C_{1} L r^{p} e^{-\lambda\left(t-r-t_{0}\right)} .
\end{aligned}
$$

By virtue of Burkholder-Davis-Gundy inequality, $\left(A_{1}\right)$, and (56), we have

$$
\begin{aligned}
& E\left(\sup _{0 \leq s \leq r} \int_{t}^{t+s}\left|g\left(X_{u}, u\right)\right| d B(u)\right) \\
& \quad \leq L r^{(p / 2)-1} \int_{t}^{t+r} \sup _{-r \leq \theta \leq 0} E|X(s+\theta)|^{p} d s \\
& \quad \leq C_{1} C(p) L r^{p / 2} e^{-\lambda\left(t-r-t_{0}\right)},
\end{aligned}
$$

where $C(p)$ is a positive constant depending on $p$ only. Thanks to $\left(A_{2}\right)$ and $(56)$, we see that

$$
\begin{aligned}
& E\left(\sum_{t \leq t_{k} \leq t+r}\left|I_{k}\left(X\left(t^{-}\right), X\left(t-d_{k}\right)^{-}\right)\right|\right)^{p} \\
& \quad \leq l_{1}^{p} E \sup _{t \leq t_{k} \leq t+r}\left|I_{k}\left(X\left(t^{-}\right), X\left(t-d_{k}\right)^{-}\right)\right|^{p} \\
& \leq l_{1}^{p} 2^{p-1} C_{1} \bar{h} e^{\lambda d} e^{-\lambda\left(t-r-t_{0}\right)} .
\end{aligned}
$$

Substituting (58)-(60) into (57) gives that

$$
E\left(\sup _{0 \leq s \leq r}|X(t+s)|^{p}\right) \leq C_{2} e^{-\lambda t}
$$

where $C_{2}$ is a positive constant. Then for all $\varepsilon \in(0, \lambda)$ and $n \in \mathbb{N}$, we have

$$
P\left(\omega: \sup _{0 \leq s \leq r}|X(n r+s)|^{p}>e^{-(\lambda-\varepsilon) n r}\right) \leq C_{2} e^{-\varepsilon n r} .
$$


Using the Borel-Cantelli Lemma, we see that there exists an $n_{0}(\omega)$ such that, for almost all $\omega \in \Omega, n \geq n_{0}(\omega)$,

$$
\sup _{0 \leq s \leq r}|X(t+s)|^{p} \leq e^{-(\lambda-\varepsilon) n r},
$$

where $n r \leq t \leq(n+1) r$. It follows that

$$
\limsup _{n \rightarrow \infty} \frac{\operatorname{logsup}_{n r \leq t \leq(n+1) r}|X(t)|}{(n+1) r} \leq \frac{-(\lambda-\varepsilon)}{p} \text {, a.s. }
$$

Consequently,

$$
\limsup _{t \rightarrow \infty} \frac{\log |X(t)|}{t} \leq \frac{-(\lambda-\varepsilon)}{p} \text {, a.s. }
$$

Let $\varepsilon \rightarrow 0$; then the result follows.

\section{Numerical Examples}

In this section, two numerical examples are given to show the effectiveness of the main results derived in the preceding section.

Example 8. Consider a stochastic delay differential system with delayed impulses as follows:

$$
\begin{array}{r}
d X(t)=[0.5 X(t)+0.125 X(t-0.2)] d t \\
+0.5 X(t-0.2) d B(t), \quad t \neq t_{k}, \quad t \geq t_{0}, \\
\Delta X\left(t_{k}\right)=-0.7 X\left(t_{k}^{-}\right)+0.2 X\left(\left(t_{k}-0.6\right)^{-}\right), \\
k \in \mathbb{N}, \\
X(0)=1.2 ; \quad X(\theta)=0, \quad-0.6 \leq \theta<0,
\end{array}
$$

where $t_{k}-t_{k-1}=0.3$. Let $p=2, V(t, x)=x^{2}, c_{1}=c_{2}=1$, and $q=4$. Then

$$
\begin{aligned}
E \mathscr{L} V(t, x)= & E|X(t)|^{2}+0.25 E X(t) X(t-0.2) \\
& +0.25 E|X(t-0.2)|^{2} \\
\leq & 1.5 E|X(t)|^{2}+0.75 q E|X(t-0.2)|^{2} \\
\leq & 4.5 E|X(t)|^{2} .
\end{aligned}
$$

Choose $\gamma_{1}=4.5, \gamma=0.265, \beta_{1}=0.2, d=0.6, \rho_{1 k}=0.18$, $\rho_{2 k}=0.08, \eta_{3 k-2}=1 / 2, \eta_{3 k-1}=1 / 2, \eta_{3 k}=4, \lambda=0.1$, and $\bar{h}=$ $L=1$. Clearly, $\left(\mathrm{A}_{1}\right)$ and $\left(\mathrm{A}_{2}\right)$ hold, and $q>\left(e^{\lambda r} / \gamma\right)=2.082$, $\gamma=0.265<e^{-\left(\gamma_{1}+\lambda\right) \beta_{2}}=0.316$. Thus, by Theorems 3 and 7 the trivial solution of system (66) is $p$ th moment and an almost sure exponential stability.

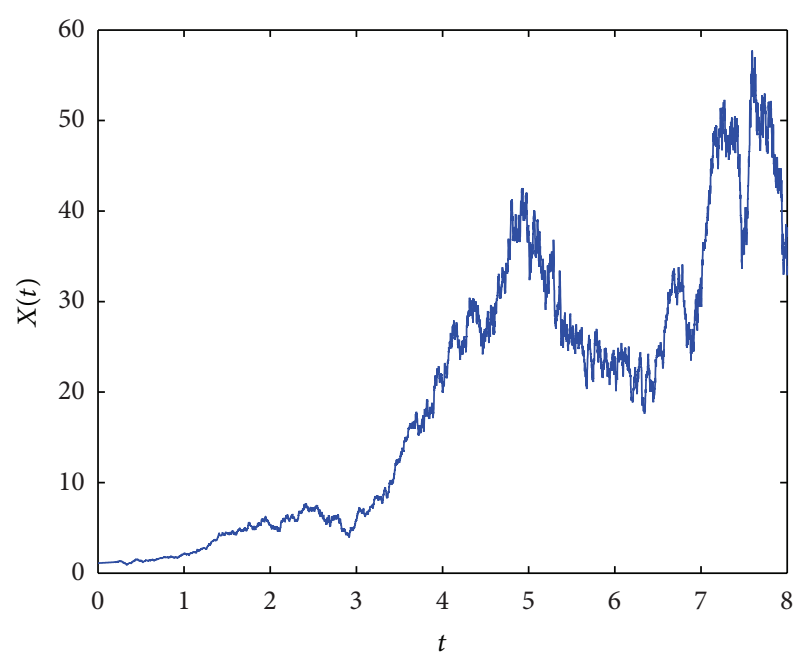

FIgURE 1: System without impulses for Example 1.

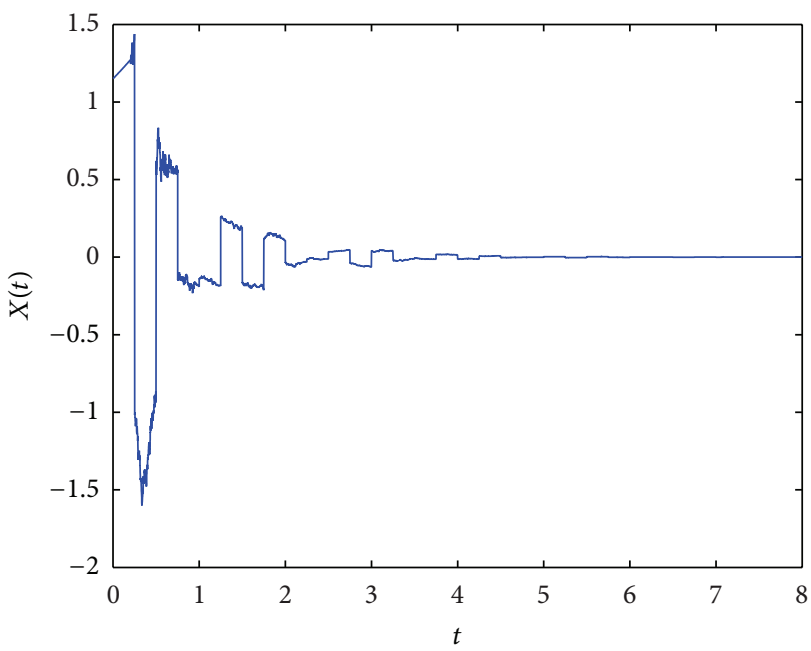

FIgURE 2: System with impulses for Example 1.

It can be seen in Figures 1 and 2 that unstable continuous dynamics of system (66) can be successfully stabilized by delayed impulses.

Example 9. Consider a stochastic delay differential system with delayed impulses as follows

$$
\begin{aligned}
& d X(t)=[-0.9 X(t)+0.125 X(t-1)] d t \\
& \quad+0.5 X(t-1) d B(t), \quad t \neq t_{k}, \quad t \geq t_{0}, \\
& \begin{aligned}
& \Delta X\left(t_{k}\right)=-0.5 X\left(t_{k}^{-}\right) \\
&+0.2 X\left(\left(t_{k}-2\right)^{-}\right), \quad k \in \mathbb{N}, \\
& X(0)=-1 ; \quad X(\theta)=0, \quad-2 \leq \theta<0,
\end{aligned}
\end{aligned}
$$




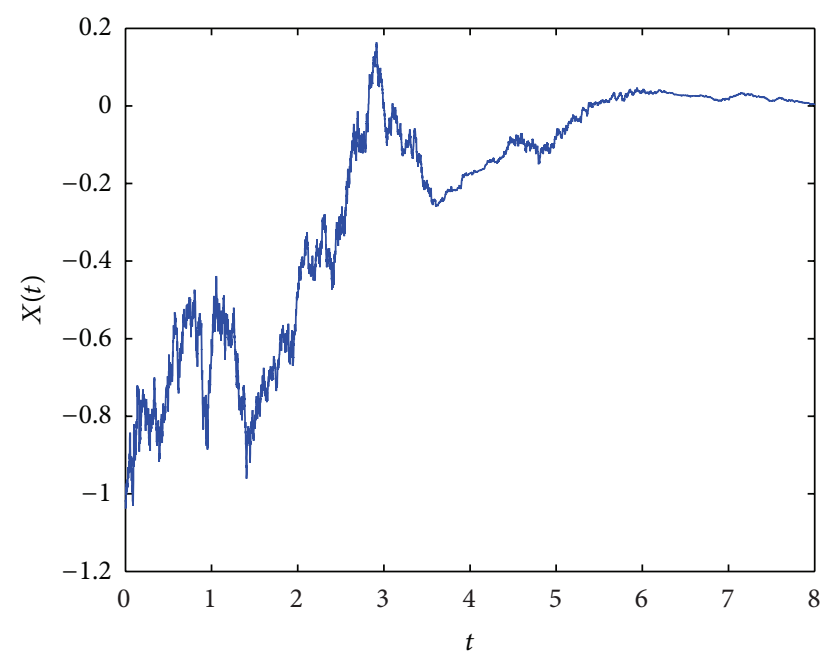

FIGURE 3: System without impulses for Example 2.

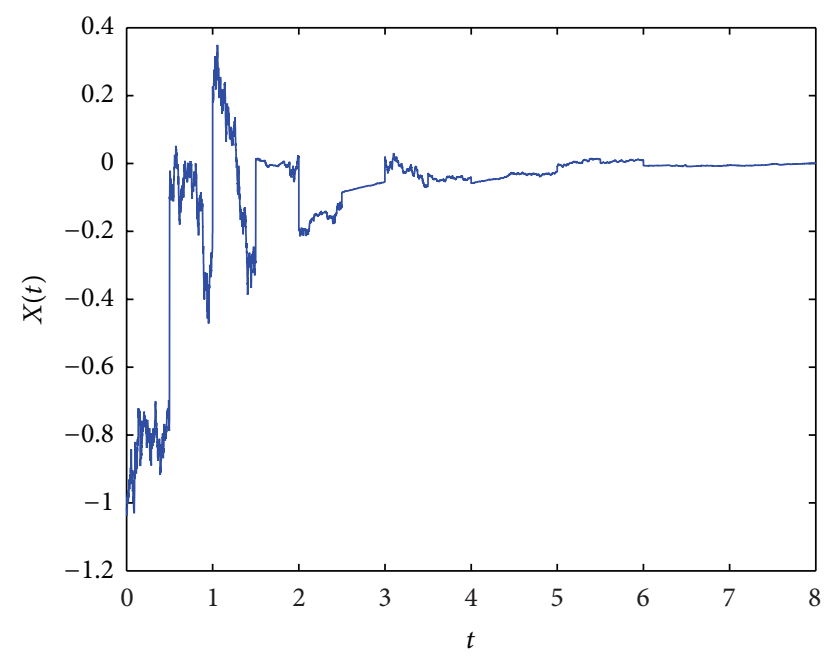

FIGURE 4: System with impulses for Example 2.

where $t_{k}-t_{k-1}=0.5$. Let $p=2, V(t, x)=x^{2}, c_{1}=c_{2}=1$, and $q=4 / 3$; then

$$
\begin{aligned}
E \mathscr{L} V(t, x)= & -1.8 E|X(t)|^{2}+0.25 E X(t) X(t-1) \\
& +0.25 E|X(t-1)|^{2} \\
\leq & -1.3 E|X(t)|^{2}+\frac{3 q}{4} E|X(t)|^{2} \\
= & -0.3 E|X(t)|^{2} .
\end{aligned}
$$

Choose $\gamma_{2}=0.3, \beta_{1}=0.5, d=0.6, \rho_{1 k}=0.5, \rho_{2 k}=0.08$, $\lambda=0.1, \bar{h}=1$, and $L=1.2$. Therefore, $\left(\mathrm{A}_{1}\right)$ and $\left(\mathrm{A}_{2}\right)$ hold, and $\max _{k \in \mathbb{N}}\left\{\left(\rho_{1 k} / \min \left\{q e^{-\lambda r}, e^{\left(\gamma_{2}-\lambda\right) \beta_{1}}\right\}\right)+\rho_{2 k} e^{\lambda d}\right\}=0.651<1$ and $q=4 / 3>e^{\lambda r}=1.106$. Thus, by Theorems 5 and 7 the trivial solution of system (68) is $p$ th moment and an almost sure exponential stability.

It can be seen from Figures 3 and 4 that the delayed impulses can robust the stability of the system (68).

\section{Conclusion}

The pth moment and almost sure exponential stability are investigated in this paper. Using Razumikhin methods, several sufficient conditions are established for stability of stochastic delay differential systems with delayed impulses. Finally, two numerical simulation examples are offered to verify the effectiveness of the main results.

\section{Conflict of Interests}

The author declares that there is no conflict of interests regarding the publication of this paper.

\section{Acknowledgment}

The project was sponsored by the Natural Science Foundation of China (no. 11326121), the Anhui Excellent Youth Fund (2013SQRL033ZD), and the Natural Science Foundation of Anhui Province (Grant no. 1408085QA09).

\section{References}

[1] V. Lakshmikantham, D. D. Baĭnov, and P. S. Simeonov, Theory of Impulsive Differential Equations, World Scientific Publishing, Singapore, 1989.

[2] X. Liu, "Stability results for impulsive differential systems with applications to population growth models," Dynamics and Stability of Systems, vol. 9, no. 2, pp. 163-174, 1994.

[3] T. Yang, Impulsive Systems and Control: Theory and Applications, Nova Science, New York, NY, USA, 2001.

[4] J. P. Hespanha, D. Liberzon, and A. R. Teel, "Lyapunov conditions for input-to-state stability of impulsive systems," Automatica, vol. 44, no. 11, pp. 2735-2744, 2008.

[5] P. Naghshtabrizi, J. P. Hespanha, and A. R. Teel, "Exponential stability of impulsive systems with application to uncertain sampled-data systems," Systems \& Control Letters, vol. 57, no. 5, pp. 378-385, 2008.

[6] D. D. Bainnov and P. S. Simeonov, Systems with Impulse Effect: Stability, Theory and Applications, Ellis Horwood, Chichester, UK, 1989.

[7] Z. Li, C. B. Soh, and X. Xu, "Stability of impulsive differential systems," Journal of Mathematical Analysis and Applications, vol. 216, no. 2, pp. 644-653, 1997.

[8] C. Li, L. Chen, and K. Aihara, "Impulsive control of stochastic systems with applications in chaos control, chaos synchronization, and neural networks," Chaos, vol. 18, no. 2, Article ID 023132, 11 pages, 2008.

[9] H. Wu and J. Sun, " $p$-moment stability of stochastic differential equations with impulsive jump and Markovian switching," Automatica, vol. 42, no. 10, pp. 1753-1759, 2006.

[10] B. Liu, "Stability of solutions for stochastic impulsive systems via comparison approach," IEEE Transactions on Automatic Control, vol. 53, no. 9, pp. 2128-2133, 2008.

[11] S. Peng and B. Jia, "Some criteria on pth moment stability of impulsive stochastic functional differential equations," Statistics \& Probability Letters, vol. 80, no. 13-14, pp. 1085-1092, 2010.

[12] P. Cheng and F. Deng, "Global exponential stability of impulsive stochastic functional differential systems," Statistics \& Probability Letters, vol. 80, no. 23-24, pp. 1854-1862, 2010. 
[13] J. Liu, X. Liu, and W.-C. Xie, "Impulsive stabilization of stochastic functional differential equations," Applied Mathematics Letters, vol. 24, no. 3, pp. 264-269, 2011.

[14] L. Pan and J. Cao, "Exponential stability of impulsive stochastic functional differential equations," Journal of Mathematical Analysis and Applications, vol. 382, no. 2, pp. 672-685, 2011.

[15] S. Peng and Y. Zhang, "Razumikhin-type theorems on $p$ th moment exponential stability of impulsive stochastic delay differential equations," IEEE Transactions on Automatic Control, vol. 55, no. 8, pp. 1917-1922, 2010.

[16] N. van de Wouw, P. Naghshtabrizi, M. B. G. Cloosterman, and J. P. Hespanha, "Tracking control for sampled-data systems with uncertain time-varying sampling intervals and delays," International Journal of Robust and Nonlinear Control, vol. 20, no. 4, pp. 387-411, 2010.

[17] X. Liu, X. Shen, Y. Zhang, and Q. Wang, "Stability criteria for impulsive systems with time delay and unstable system matrices," IEEE Transactions on Circuits and Systems I. Regular Papers, vol. 54, no. 10, pp. 2288-2298, 2007.

[18] A. Khadra, X. Z. Liu, and X. ShermanShen, "Analyzing the robustness of impulsive synchronization coupled by linear delayed impulses," IEEE Transactions on Automatic Control, vol. 54, no. 4, pp. 923-928, 2009.

[19] W.-H. Chen and W. X. Zheng, "Exponential stability of nonlinear time-delay systems with delayed impulse effects," Automatica, vol. 47, no. 5, pp. 1075-1083, 2011.

[20] P. Cheng, F. Deng, and F. Yao, "Exponential stability analysis of impulsive stochastic functional differential systems with delayed impulses," Communications in Nonlinear Science and Numerical Simulation, vol. 19, no. 6, pp. 2104-2114, 2014.

[21] F. Yao, F. Deng, and P. Cheng, "Exponential stability of impulsive stochastic functional differential systems with delayed impulses," Abstract and Applied Analysis, vol. 2013, Article ID 548712, 8 pages, 2013. 


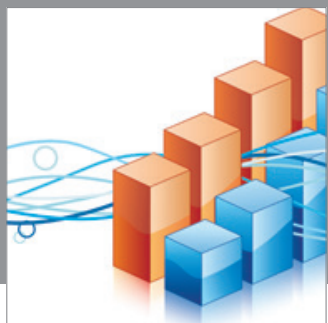

Advances in

Operations Research

mansans

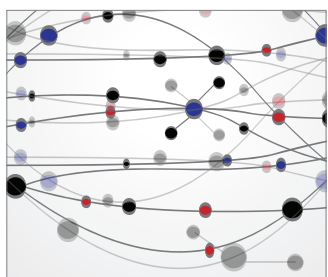

The Scientific World Journal
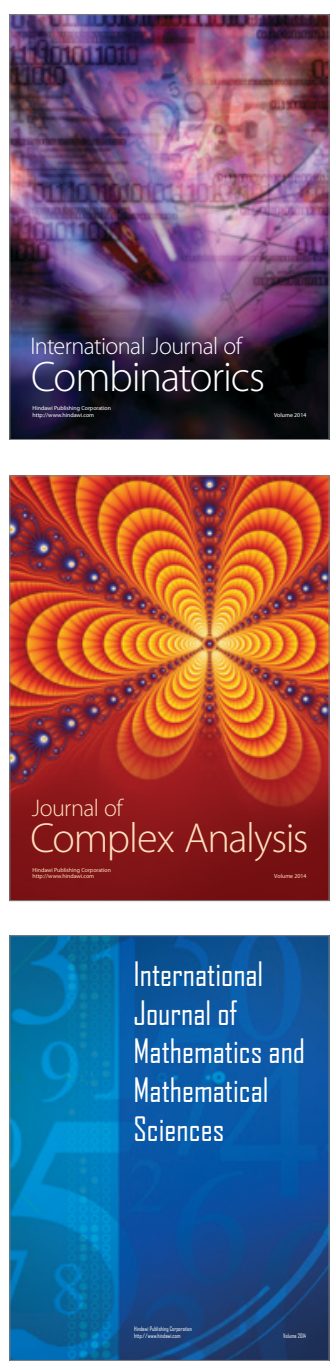
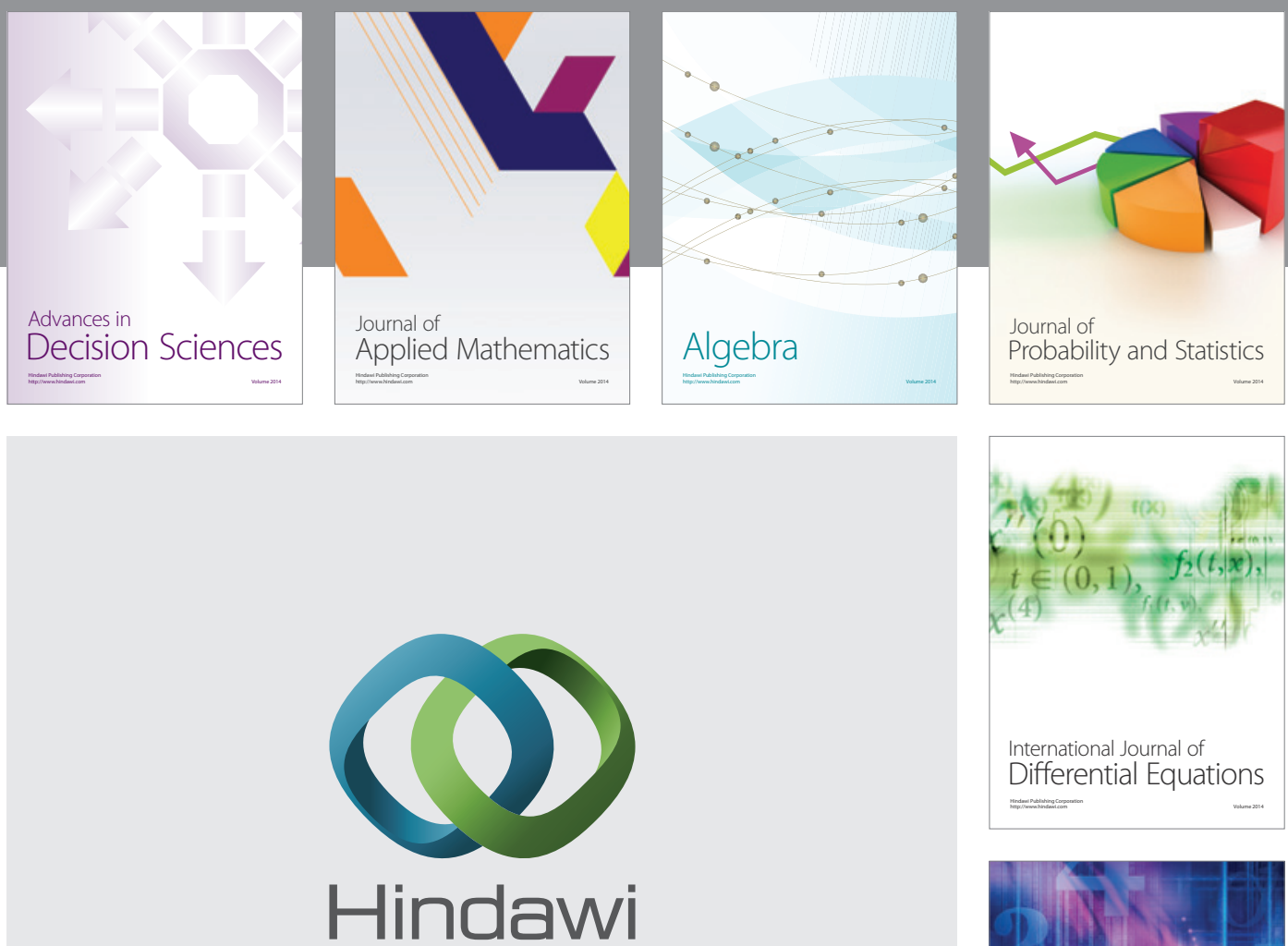

Submit your manuscripts at http://www.hindawi.com
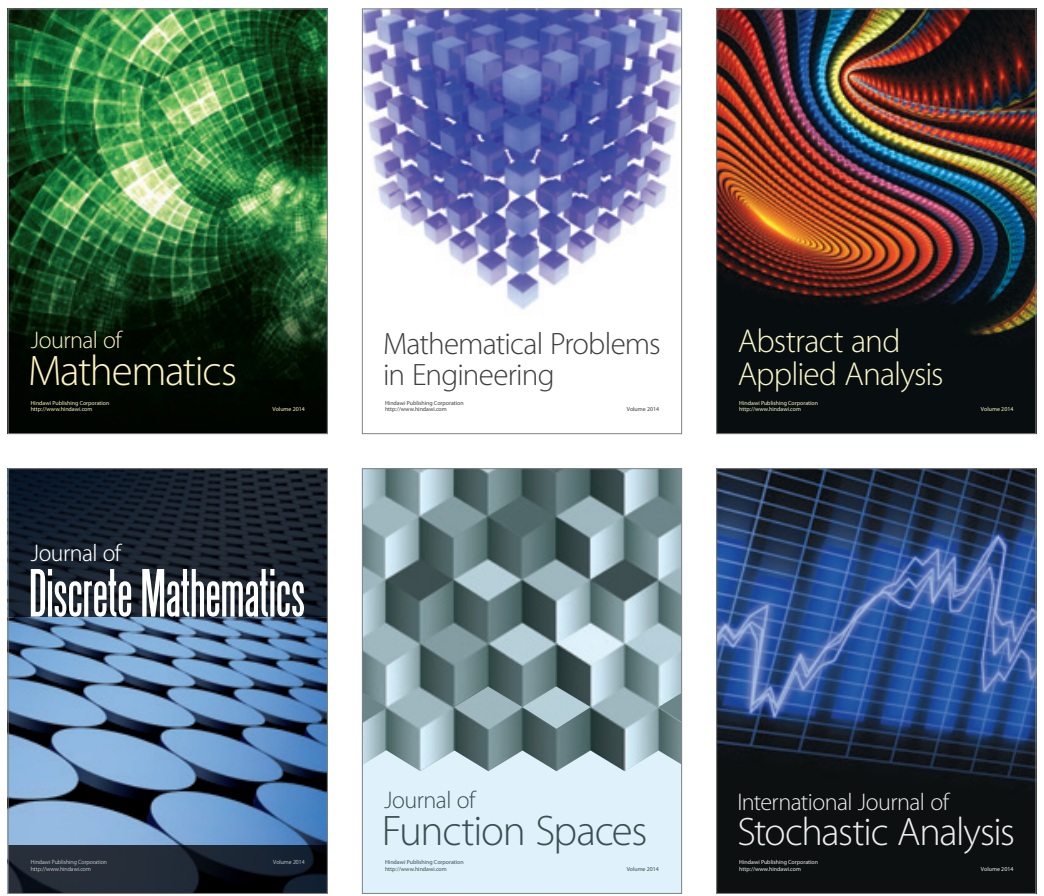

Journal of

Function Spaces

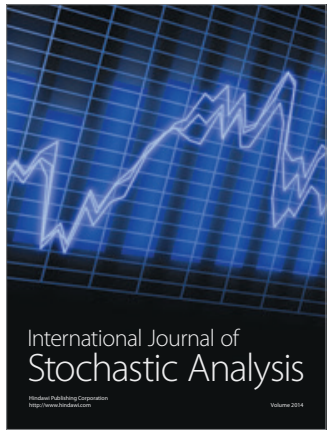

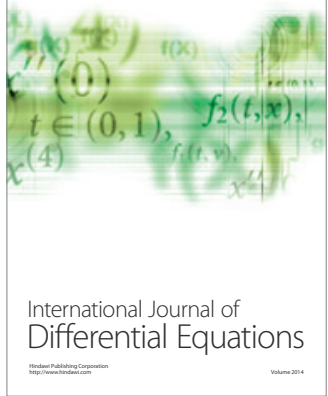
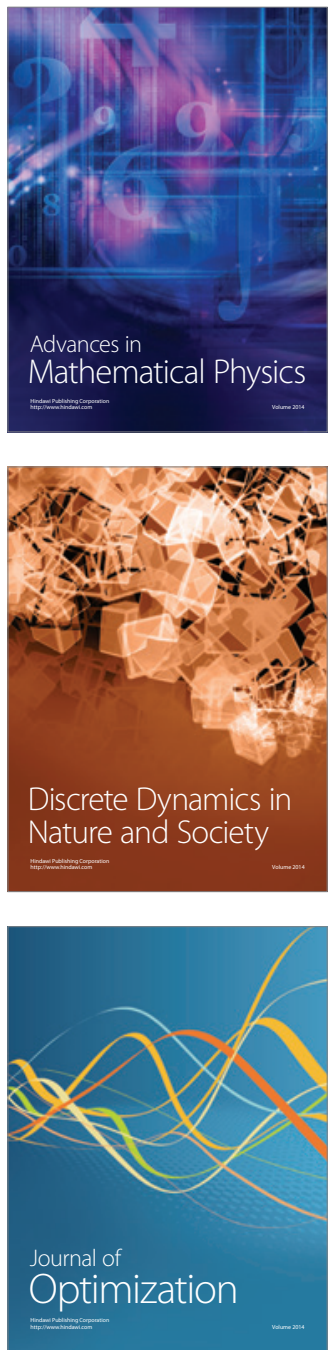